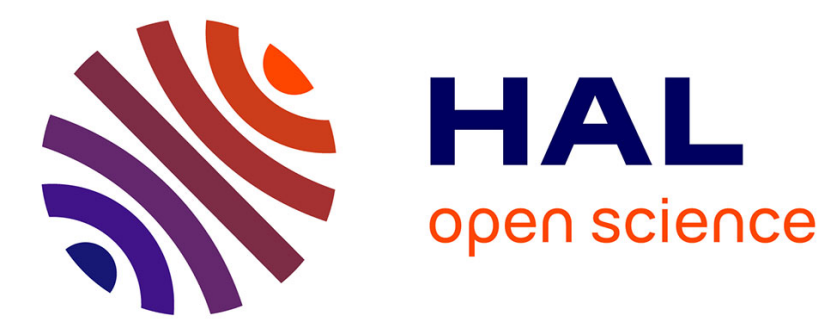

\title{
Mourir d'amour
}

Isée Bernateau

\section{To cite this version:}

Isée Bernateau. Mourir d'amour. Adolescence, 2010, Et la mort?, 2 (72), pp.281 - 287. 10.3917/ado.072.0281 . hal-01509877

\section{HAL Id: hal-01509877 https://hal.science/hal-01509877}

Submitted on 18 Apr 2017

HAL is a multi-disciplinary open access archive for the deposit and dissemination of scientific research documents, whether they are published or not. The documents may come from teaching and research institutions in France or abroad, or from public or private research centers.
L'archive ouverte pluridisciplinaire HAL, est destinée au dépôt et à la diffusion de documents scientifiques de niveau recherche, publiés ou non, émanant des établissements d'enseignement et de recherche français ou étrangers, des laboratoires publics ou privés. 


\section{MOURIR D'AMOUR}

\section{Isée Bernateau}

\section{L’Esprit du temps | «Adolescence »}

$2010 / 2 n^{\circ} 72 \mid$ pages 281 à 287

ISSN 0751-7696

ISBN 9782847951745

Article disponible en ligne à l'adresse :

http://www.cairn.info/revue-adolescence1-2010-2-page-281.htm

\section{Pour citer cet article :}

Isée Bernateau, " Mourir d'amour », Adolescence 2010/2 (n 72), p. 281-287. DOI 10.3917/ado.072.0281

Distribution électronique Cairn.info pour L'Esprit du temps.

(C) L'Esprit du temps. Tous droits réservés pour tous pays.

La reproduction ou représentation de cet article, notamment par photocopie, n'est autorisée que dans les limites des conditions générales d'utilisation du site ou, le cas échéant, des conditions générales de la licence souscrite par votre établissement. Toute autre reproduction ou représentation, en tout ou partie, sous quelque forme et de quelque manière que ce soit, est interdite sauf accord préalable et écrit de l'éditeur, en dehors des cas prévus par la législation en vigueur en France. Il est précisé que son stockage dans une base de données est également interdit. 


\title{
MOURIR D'AMOUR |
}

\author{
ISÉE BERNATEAU
}

« Mourir d'amour » : le lien entre l'amour et la mort n'est jamais aussi présent que pendant l'adolescence, où les premières amours sont toujours susceptibles d'ouvrir sur un tragique morbide. Éros et Thanatos, au lieu de lutter l'un contre l'autre, viennent parfois se rencontrer, et même se confondre. Y aurait-il dans le sentiment amoureux quelque chose qui se rapproche de la mort ? Ou faudrait-il plutôt considérer que la mort est convoquée par l'adolescent pour contre-investir une pulsionnalité dont le surgissement est traumatique?

En 1836, un an après avoir écrit son grand drame politique La Mort de Danton, le dramaturge allemand $\mathrm{G}$. Büchner rédige une petite pièce de théâtre intitulée Léonce et Léna. Cette comédie, surprenante à bien des égards, renouvelle considérablement le genre. L'intrigue est pourtant très simple : le prince héritier Léonce, qui ne voit aucun sens à sa vie, s'enfuit en Italie pour échapper à son mariage avec Léna, arrangé par son père. De son côté, la princesse Léna, promise à Léonce qu'elle ne connaît pas, fuit également en Italie. Le hasard fait qu'ils se rencontrent et tombent amoureux, pour finalement se marier comme prévu, tout en croyant échapper à leur destinée. La pièce de G. Büchner s'inscrit dans un contexte politique de désenchantement qui succède à la Révolution française et qui voit un ordre réprouvé et considéré comme caduc continuer malgré tout à régenter la société. Ainsi, au-delà de son intrigue de comédie, elle a la particularité de mettre en scène un désœuvrement et un désespoir proprement adolescents. Léonce et Léna, héros sans envie ni projet, incarnent sur la scène un mal-être qui va devenir paradigmatique, 
selon l'expression de Ph. Gutton, d'une certaine dépressivitél propre à cet âge. Ils sont des figures privilégiées de ce désenchantement romantique, désenchantement dont la symptomatologie continue encore aujourd'hui de nourrir le mal de vivre adolescent. Ils sont de ce point de vue les ancêtres de nos adolescents déprimés, qui leur empruntent certains traits et certaines attitudes. Le caractère négatif de leur investissement, à la fois d'eux-mêmes et du monde, est caractéristique d'un mode d'être adolescent qui refuse et rejette le monde adulte dans lequel ils doivent pourtant s'inscrire. Ces adolescents font notamment état d'un type particulier de désinvestissement, d'une certaine apologie du vide et du rien, qui n'est d'ailleurs pas sans susciter dans leur entourage une certaine fascination. Mais de quel mal souffrent-ils ? Auraient-il peur du désir?

Dans un premier temps, la relation amoureuse entre Léonce et Léna est compliquée, voire empêchée par d'innombrables entraves qui retardent à l'envi leur rencontre. Mais les obstacles qui s'opposent à leur union ne sont pas, comme dans la comédie classique, vecteurs d'un désir que rien ne pourrait arrêter. C'est même le contraire : l'obstacle du mariage arrangé existe mais il n'est qu'un prétexte ; les héros, dans leur ennui et leur inertie, n'auront aucun mal à déjouer la volonté paternelle, elle-même peu assurée d'ailleurs. Le mariage de Léonce et Léna se fera ! Et pourtant... la rencontre avec l'objet d'amour, quand elle a enfin lieu, s'avère traumatique pour eux, et la mort, ou le risque de mort, apparaît là où l'on serait en droit d'attendre l'expression d'un désir amoureux. Leur première rencontre se fait sous le signe de la mort et de la dépression. Léonce est en proie à une rêverie mélancolique quand la voix de Léna lui répond et se fait l'écho de sa tristesse :

"- Léonce, perdu dans ses rêves: Tous les chemins sont longs! Le battement de l'horloge de la mort dans notre poitrine est lent, et chaque goutte de sang prend son temps, et notre vie est une fièvre qui n'en finit pas. Pour des pieds fatigués, tout chemin est trop long...

- Léna, qui l'écoute craintive et songeuse : Et pour des yeux fatigués, toute lumière trop violente, et pour des lèvres fatiguées, tout souffle trop lourd (souriant), et pour des oreilles fatiguées, toute parole de trop. (Elle entre dans la maison avec la gouvernante) $»^{2}$.

1. Gutton, 1986, p. 172 : « La dépression donnerait des représentations à l'affect dépressif ; elle signale le travail de représentativité, la figuration du vide. Le Moi y convoquerait stratégiquement l'objet représentable pour éviter la dépressivité. Lorsque le dépressif représente, il devient déprimé. »

2. Büchner G. (1836). Léonce et Léna. In : Euvres complètes, inédits et lettres. Paris : Seuil, 1988, pp. 199-232. 
L'affect dépressif traverse ces deux répliques, et Léna identifie d'ailleurs dans la scène suivante Léonce comme un être «malheureux, irrémédiablement ». Pourtant, Léonce, après avoir entendu Léna, sent sa mélancolie le quitter, car la certitude d'avoir été compris le libère de son oppression intérieure. On pourrait donc penser que la découverte du sentiment amoureux, inconnu jusqu'ici pour Léonce, opère comme un charme magique et le délivre de son commerce avec la mort présent depuis le début de la pièce. Mais il n'en est rien. Dès la deuxième rencontre, une analogie s'opère pour Léonce et Léna entre l'amour et la mort, et leur dialogue amoureux se fait rêverie d'un baiser à la morte :

«-Léna, se parlant à elle-même : La fauvette a gazouillé en rêve, la nuit dort plus profondément, ses joues sont plus pâles, et son souffle plus paisible. La lune est comme un enfant endormi, dans son sommeil ses boucles dorées sont retombées sur son visage...Ô son sommeil est la mort. L'ange mort repose sur son oreiller d'obscurité et les étoiles brûlent autour de lui comme des cierges. Pauvre enfant, les hommes noirs ne vont-ils pas bientôt venir te chercher? Où est ta maman ? Ne veutelle pas t'embrasser encore une fois ? Quelle tristesse, mort et si seul.

- Léonce : Lève-toi dans ta robe blanche et marche derrière le cadavre dans la nuit et chante-lui le chant des morts $»^{3}$.

Si l'enfant mort est par excellence le fantasme du mélancolique, l'enfant endormi, que « la mère tient et retient endormi parce qu'elle ne saurait, d'une quelconque façon, survivre à sa perte $»^{4}$ est sans doute, comme P. Fédida le propose, celui du dépressif. Or, dans notre scène, l'enfant endormi devient cadavre : l'oscillation entre la dépression et la mélancolie est donc patente, mais c'est autour de la question de la perte et de la solitude que les choses s'organisent. La rencontre amoureuse fait surgir le fantasme de l'enfant mort et abandonné, comme si l'investissement libidinal de l'objet venait mettre à mort l'enfance en séparant l'enfant de sa mère. Léonce et Léna semblent tenter de conjurer le risque d'une perte, et l'enfant mort est le signe, l'incarnation imaginaire de cette perte qui leur est insupportable car elle est perte d'eux-mêmes.

Aimer un mort, remplacer un mort, faire le mort, être mort : le héros büchnérien a la tentation du suicide, comme si embrasser le néant « pour de vrai » était l'aboutissement de cette quête désespérée qui le conduit à l'approcher sans cesse comme pour renouer avec l'objet perdu. Le départ précipité de Léonce et de Léna pour l'Italie résonne comme une rupture violente avec ce qui a précédé, métaphore d'un suicide à soi-même et au

3. Ibid., p. 217.

4. Fédida, 1978, p. 153. 
monde. Une telle analogie se vérifie puisque Léonce envisage de se suicider juste après sa première rencontre avec Léna : «Ah ! c’est trop ! Beaucoup trop ! Tout mon être est dans cet instant. Meurs à présent. Plus c'est impossible. Comme elle est fraîche, étincelante de beauté la création qui s'arrache au chaos et s'avance vers moi. La terre est un calice d'or sombre, la lumière y bouillonne, déborde, et les étoiles en jaillissent. Mes lèvres tètent goulûment : cette goutte de félicité fait de moi un précieux récipient. Adieu, sainte coupe ! (Il veut se jeter dans la rivière) $\gg$. Dans cette tirade, Léonce fait l'expérience d'une rencontre amoureuse orgasmique avec la nature, métaphorique du désir charnel qu'il a ressenti pour Léna. Mais cet épanchement le conduit au suicide, qui fonctionne alors comme un équivalent de la complétude ressentie. Il s'agit, pour Léonce, de ne pas éprouver la déperdition entraînée par la fin de ce sentiment d'union charnelle avec l'univers, et de ne pas se retrouver séparé de nouveau de luimême et du monde, en proie à son solipsisme destructeur.

Face à Rosetta, une jeune fille qui se meurt d'amour pour lui, Léonce affirme, non sans cruauté : « Ô, un amour qui se meurt est plus beau qu'un amour qui vient de naître. Je suis un Romain ; pour achever ce festin grandiose, au dessert, les poissons dorés font chatoyer les couleurs de l'agonie. Comme le rouge s'éteint sur ses joues, comme ses yeux doucement perdent leur éclat, comme ses gestes délicatement s'amplifient et retombent ! Adio, adio, mon amour, j'aimerai ton cadavre $»^{6}$. Aimer un cadavre, ne serait-ce pas en effet le moyen de « refroidir » un désir ressenti comme trop menaçant pour le narcissisme du sujet, puisqu'il lui intime l'ordre de se déprendre d'objets vécus comme partie intégrante de lui-même? Dans cette optique, l'obsession de la mort peut être comprise comme une tentative de se décoller d'une union incestueuse vécue comme dangereuse et mortifère. Mettre la mort à la place de l'amour serait alors, non seulement la conséquence d'une dépression liée à la perte de l'objet, mais aussi, et dans le même mouvement, le moyen de donner figuration à un éprouvé libidinal qui, sinon, serait trop proche d'un irreprésentable incestueux :

5. Büchner, Léonce et Léna. Op. cit., pp. 217-218.

6. Ibid., p. 205. 
«-Léna: Qui parle?

- Léonce: Un rêve.

- Léna : Les rêves sont bienheureux.

- Léonce: Soit, rêve-toi bienheureuse et laisse-moi être ton rêve bienheureux.

- Léna : La mort est le rêve bienheureux par excellence.

- Léonce : Soit, laisse-moi être ton ange de la mort. Laisse mes lèvres se poser comme des ailes sur tes yeux. (Il l'embrasse). Beau cadavre, tu reposes sur le linceul noir de la nuit avec tant de grâce que la nature se met à haïr la vie et devient amoureuse de la mort.

- Léna : Non, laisse-moi. (Elle se lève d'un bond et s'en va rapidement)»7.

En se faisant ange de mort, Léonce tente d'échapper au danger contenu dans la promesse de l'union charnelle avec Léna, promesse qui, si elle se réalise, menace également l'intégrité de son être. Dans ce refus de faire corps avec l'objet, qui s'actualise en attaque perverse de l'autre, puisque Léonce " refroidit» ses partenaires en les assimilant à des cadavres, il y a un mouvement d'évitement qui est une véritable stratégie de la négativité. De même Léna en appelle à la mort, « rêve bienheureux » qui fait taire tous les autres rêves, et qui permet de résister à la menace d'intrusion que l'objet fait peser sur le Moi. Ainsi nos deux héros, qui semblent souffrir de ne rien pouvoir aimer, sont en réalité assaillis par la menace que fait peser sur eux l'investissement sexuel de l'objet. Leur apologie du vide vient témoigner d'un trop plein, d'un envahissement d'imagos parentales excitantes et intrusives que l'évocation de la mort tente de mettre à distance. Leur affectation de la mort est le signe d'une mise en panne, d'un refus de s'investir et de s'engager dans un processus quel qu'il soit, en même temps qu'un moyen de refroidir un sexuel dont l'empreinte incestueuse est trop forte.

Quand elle devient omniprésente à l'adolescence, on peut donc penser que la mort fonctionne comme un objet contraphobique censé protéger l'adolescent de désirs dont la résonance incestueuse lui est insupportable. Le hors temps d'un univers régi par la mort offre un écran, un voile qui dissimule et refroidit cet éprouvé libidinal. À l'abri de ce voile, les désirs incestueux trouvent un exutoire car : " Seule la mort

7. Ibid., p. 217. 
possède ce caractère de "sans contenu " qui lui permet de représenter, dans l'inconscient, la réalisation du vœu incestueux, vœu équivalent à celui de posséder la virtualité de réaliser tous les désirs imaginables $\rangle^{8}$. Cette concordance formelle entre l'inceste et la mort aide à comprendre la signification orgasmique de certains suicides à l'adolescence : ils sont à la fois un moyen de se séparer imaginairement d'un objet toujours trop envahissant, mais aussi et surtout un moyen de s'unir à lui pour toujours dans la mort. Retourner vers la fusion, tel serait le désir caché sous les propos suicidaires de Léonce. Son désir de suicide ouvre sur le sentiment océanique cher à R. Rolland, d'une « absence de frontières » et d'un « lien avec le Tout $\gg$. La mort, sous couvert d'éteindre tout désir, devient alors le lieu dans lequel le plus grand des désirs serait susceptible de se réaliser.

Mais Léonce et Léna, contrairement à La Mort de Danton et à Woyzek, les deux grands drames de Büchner, est une comédie, et la pièce se clôt par un mariage. Il est vrai que nos deux héros, en revenant au palais et en concluant leur mariage, sont rattrapés par ce à quoi ils avaient tenté d'échapper. Néanmoins, le programme établi par Léonce tente d'annuler cette répétition pour promulguer un nouvel et différent état des lieux : «En fait je sais bien ce que tu veux, nous faisons détruire toutes les horloges, nous interdisons tous les calendriers et nous ne comptons plus les heures et les mois que sur l'horloge des fleurs, selon la floraison et la fructification. Et ensuite, nous entourons ce petit pays de miroirs ardents pour qu'il n'y ait plus d'hiver et qu'en été nous nous distillions jusqu'à Ischia et Capri, et que toute l'année nous soyons parmi les roses et les violettes, les orangers et les lauriers $»^{10}$. Sans doute est-il possible de lire, dans ce programme, la tentative d'une abolition du temps au profit d'un espace éternellement accueillant. Le problème est qu'un tel programme, si séduisant soit-il, ne peut que se dénoncer lui-même comme parfaitement utopique. Les « miroirs ardents » qui emmènent en Italie sont révélateurs du romantisme de Léonce et de son goût pour l'Italie douce et chaleureuse, mais ils sont surtout des fabricants d'illusion pour tenter

8. Richard, 1989, p. 39.

9. Freud, 1930, p. 253.

10. Büchner, Léonce et Léna. Op. cit., p. 225. 
d'échapper à un réel décevant. Finalement, l'illusion utopique ne renvoie que plus violemment au réel qu'elle tente de nier : nul ne peut échapper à son temps. La pièce s'interrompt dans une suspension du temps et de l'espace dont l'artificialité est proclamée et assumée par les personnages : ils ne peuvent pas vivre dans le monde tel qu'il est, mais ils ne peuvent que le critiquer, et non bouleverser véritablement l'ordre des choses. L'impasse existentielle des héros contraint la pièce à se dénoncer ellemême comme utopie, comme construction artificielle qui dénonce l'artificialité d'un système politique et social. Ainsi, Léonce et Léna, tout en évoquant le péril que constitue pour l'adolescent la rencontre de l'autre sexe, évoque également le seul lieu où cette union, des cœurs et des corps, est pour l'instant susceptible de se réaliser, le lieu de la fiction. À l'abri de la fiction, quand bien même cette fiction se dénonce comme telle, l'adolescent peut rêver, fantasmer, imaginer une jouissance à laquelle il a pour le moment peur de se confronter. Alors, comme dans Léonce et Léna, il peut se marier, mais « pour de faux ».

\section{BIBLIOGRAPHIE}

FÉDIDA P. (1978). L'Absence. Paris : Gallimard.

FREUD S. (1930). Le Malaise dans la culture. In : Euvres complètes, T. XVIII. Paris :

PUF, 1994, pp. 245-333.

GUTTON PH. (1986). Dépressivité et stratégies dépressives. Adolescence, 4 : 171-178, et

Monographie Adolescence, 1999, pp. 9-16.

RICHARD F. (1989). Psychothérapie des dépressions narcissiques. Paris : PUF.

Isée Bernateau

Centre d'Étude en Psychopathologie et Psychanalyse

Université Paris VII-Denis Diderot

UFR Sciences Humaines Cliniques

26, rue de Paradis

75010 Paris, France

isee.bernateau@orange.fr 